\title{
QUANTITATIVELY HYPER-POSITIVE REAL FUNCTIONS
}

\author{
DANIEL ALPAY AND IZCHAK LEWKOWICZ
}

\begin{abstract}
Hyper-positive real, matrix-valued, rational functions are associated with absolute stability (the Lurie problem). Here, quantitative subsets of Hyper-positive functions, related through nested inclusions, are introduced. Structurally, this family of functions turns out to be matrix-convex and closed under inversion.

A state-space characterization of these functions through a corresponding Kalman-YakubovichPopov Lemma, is given. Technically, the classical Linear Matrix Inclusions, associated with passive systems, are here substituted by Quadratic Matrix Inclusions.
\end{abstract}

AMS Classification: 34H05 47N70 93B20 93C15

Key words: absolute stability, convex invertible cones, electrical circuits, feedback loops, hyper-positive real functions, K-Y-P lemma, matrix-convex set positive real functions, state-space realization.

\section{Contents}

1. Introduction

2. Matrices whose spectrum is within a subunit Disk 2

3. Quantitatively Hyper-Bonded real rational functions 4

4. Quantitatively contractive Lyapunov inclusions 6

5. Quantitatively Hyper-Positive Real Functions 8

5.1. The Lurie Problem - absolute stability 11

5.2. Structure of $\mathcal{H} \mathcal{P}_{\eta}-13$

6. Kalman-Yakubovich-Popov Lemma for $\mathcal{H P}_{\eta}$ functions

6.1. K-Y-P for Positive functions 16

6.2. K-Y-P for Hyper-Positive functions 17

References 19

\section{INTRODUCTION}

Positive real rational functions are known to be a model for passive, continuous-time, linear, time-invariant systems, see e.g. [4, Theorem 2.6.1], [6, Section 3.18], [12, Section 6.3], [21. In [13] it was pointed that the family of $m \times m$-valued positive real rational functions, forms a cone, which is matrix-convex ${ }^{1}$ and also closed under inversion. Moreover, it was also shown that this property is maximal in the sense that if one picks up a function, which is analytic in the right half plane, but not positive real, by taking positive scaling, sums and inversion of this function along with $F(s) \equiv I_{m}$ (a zero degree positive real

Daniel Alpay thanks the Foster G. and Mary McGraw Professorship in Mathematical Sciences, which supported this research.

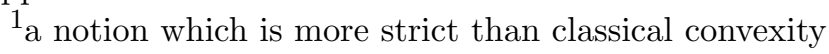


function), one can always obtain a function which is no longer analytic in the right-half plane.

It turned out that corresponding state-space realizations are also intimately linked to the structure of matrix-convex cones, closed under inversion. This can be intuitively explained by employing LMI (Linear Matrix Inclusions ${ }^{2}$ ) formulation.

In this work we focus on a dissipative subset of positive real function ${ }^{3}$ : The hyper-positive real rational functions, which are associated with absolute stability (a.k.a. the Lurie problem). We then introduce a partial ordering within this set. It turns out that here, the corresponding structure is of matrix-convex sets (not cones), closed under inversion. Furthermore here, the LMI's are substituted by Quadratic Matrix Inclusions.

The work is outlined as follows. In Sections 2 and 3 , we introduce a quantitative nested set of inclusions into the Stein matrix inclusion and of the family of Bounded real rational functions, respectively. Applying the Cayley transform, the corresponding refinement of the Riccati matrix inclusion and the Positive real rational functions, are given in Sections 4 and 5, respectively. In Section 6 a corresponding Kalman-Yakubovich-Popov Lemma is presented.

\section{Matrices Whose Spectrum is Within A SUbunit Disk}

In the sequel, we shall denote by $\overline{\mathbf{H}}_{n}\left(\mathbf{H}_{n}\right)$ the set of $n \times n$ Hermitian (non-singular) matrices. Similarly $\left(\overline{\mathbf{P}}_{n}\right) \mathbf{P}_{n}$ will be set of $n \times n$ positive (semi)-definite matrices ${ }^{4}$.

For convenience, when using the Cayley transform, we follow the (artificial yet intuitive) engineering notational distinction between $A$ (typically with spectrum in $\mathbb{C}_{R}$ ) and $\hat{A}$ (whose spectrum is typically in the unit disk).

For a given $H \in \mathbf{H}_{n}$, let us define the set of all matrices $\hat{A}$ satisfying the Stein ${ }^{5}$ inclusion,

$$
\operatorname{Stein}_{H}=\left\{\hat{A} \in \mathbb{C}^{n \times n}:\left(\begin{array}{c}
\hat{A} \\
I_{n}
\end{array}\right)^{*}\left(\begin{array}{cc}
-H & 0 \\
0 & H
\end{array}\right)\left(\begin{array}{c}
\hat{A} \\
I_{n}
\end{array}\right) \in \mathbf{P}_{n}\right\}
$$

Properties of the set $\mathbf{S t e i n}_{H}$ are explored in [15]. We now introduce a scalar parameter $\eta$, where $\eta \in(1, \infty]$, to the sets in Eq. (2.1),

$$
\operatorname{Stein}_{H}(\eta)=\left\{\hat{A} \in \mathbb{C}^{n \times n}:\left(\begin{array}{c}
\hat{A} \\
I_{n}
\end{array}\right)^{*}\left(\begin{array}{cc}
-(\eta+1) H & 0 \\
0 & (\eta-1) H
\end{array}\right)\left(\begin{array}{c}
\hat{A} \\
I_{n}
\end{array}\right) \in \mathbf{P}_{n}\right\}
$$

Note that for all $H \in \mathbf{H}_{n}$ and all $\eta \in(1, \infty]$, the matrix

$$
\left(\begin{array}{cc}
-(\eta+1) H & 0 \\
0 & (\eta-1) H
\end{array}\right)
$$

has real eigenvalues, $n$ of them are positive and $n$ negative.

Eq. (2.2) offers a more detailed examination of the set in Eq. (2.1), in the following sense,

$$
\infty>\eta>\eta_{1}>1 \Longrightarrow \operatorname{Stein}_{H}\left(\eta_{1}\right) \subset \operatorname{Stein}_{H}(\eta) \subset \operatorname{Stein}_{H},
$$

\footnotetext{
${ }^{2}$ meaning "inclusion within the set of positive (semi)-definite matrices" which is more accurate than mere "inequality".

${ }^{3}$ In fact, a proper subset of Strictly Positive Real functions.

${ }^{4}$ Recall, $\bar{X}$ denotes the closure of a set $X$.

${ }^{5}$ In some engineering circles referred to a "discrete-time Lyapunov".
} 
and

$$
\lim _{\eta \rightarrow \infty} \operatorname{Stein}_{H}(\eta)=\operatorname{Stein}_{H}
$$

Here are three basic properties of this set.

Proposition 2.1. For parameters $H \in \mathbf{H}_{n}$ and $\eta \in(1, \infty]$, the matricial set $\mathbf{S t e i n}_{H}(\eta)$ in Eq. 2.2, satisfies the following,

(i) The set $\operatorname{Stein}_{H}(\eta)$ is open ${ }^{6}$ and in particular contains all elements of the form $\lambda I_{n}$, where $\lambda \in \mathbb{C}$ and $\sqrt{\frac{\eta-1}{\eta+1}}>|\lambda|$.

(ii) This set is closed under multiplication by complex scalars of bounded modulus, i.e.

$$
\left.\hat{A} \in \operatorname{Stein}_{H}(\eta) \quad \begin{array}{c}
\lambda \in \mathbb{C} \\
\sqrt{\frac{\eta-1}{\eta+1}}>|\lambda|
\end{array}\right\} \quad \Longrightarrow \quad \lambda \hat{A} \in \operatorname{Stein}_{H}(\eta) .
$$

(iii) This set is matrix-product-contractive, i.e.

$$
\hat{A}_{a}, \hat{A}_{b} \in \operatorname{Stein}_{H}(\eta) \quad \Longrightarrow \quad\left(\hat{A}_{a} \hat{A}_{b}\right) \in \operatorname{Stein}_{H}\left(\eta_{1}\right) \quad \text { where } \quad \eta_{1}=\frac{1}{2}\left(\eta+\frac{1}{\eta}\right) .
$$

The above properties are easy to verify.

In [5, Theorem 3.5] T. Ando showed that that items (i), (ii), (iii) in Proposition 2.1 are three out of seven items characterizing any set of the form Stein $_{H}$ (without specifying $H)$. The details are technically demanding and thus omitted.

In the rest of this section we shall confine the discussion to $H \in \mathbf{P}_{n}$. Then, by multiplying Eq. (2.2), by $H^{-\frac{1}{2}}$ from both sides, one can equivalently write

$$
\operatorname{Stein}_{H}(\eta)=\left\{\hat{A} \in \mathbb{C}^{n \times n}: \sqrt{\frac{\eta-1}{\eta+1}}>\left\|H^{\frac{1}{2}} \hat{A} H^{-\frac{1}{2}}\right\|_{2}\right\}
$$

where \|\|$_{2}$ denotes the spectral (a.k.a. Euclidean) norm. As a motivation, one has the following model of a stability robustness problem.

\section{Difference inclusion stability problem}

Let $x(\cdot)$ be a real vector-valued sequence satisfying,

$$
x(k+1)=A(k, x(k)) x(k) \quad k=0,1,2, \ldots
$$

where the actual sequence $\{A(0, x(0)), A(1, x(1)), A(2, x(2)), \ldots\}$ can be arbitrary.

Proposition 2.2. If in the above difference inclusion,

$$
A(\cdot, \cdot) \in \operatorname{Stein}_{I_{n}}(\eta)
$$

then,

$$
\|x(0)\|_{2} \cdot\left(\frac{\eta-1}{\eta+1}\right)^{\frac{k}{2}} \geq\|x(k)\|_{2} \quad \forall x(0) \quad k=0,1,2, \ldots,
$$

The restriction in Eq. 2.3 of the set $\operatorname{Stein}_{H}(\eta)$ to $H \in \mathbf{P}$, enables one to write Eq. 2.3 and thus introduce an additional property. To describe it, we need the following.

Definition 2.3. A set $\mathbf{A}$ of $n \times n$ matrices is called matrix-convex if for all natural $k$ and all $n \times n k$ isometry $\Upsilon=\left(\begin{array}{c}v_{1} \\ \vdots \\ v_{k}\end{array}\right)$ i.e. $I_{n}=\Upsilon^{*} \Upsilon=\sum_{j=1}^{k} v_{j}^{*} v_{j}$ one has that whenever the

\footnotetext{
${ }^{6}$ In the sense that $\overline{\mathbf{P}}_{n}$ is the closure of $\mathbf{P}_{n}$.
} 
matrices $A_{1}, \ldots, A_{k}$ are in $\mathbf{A}$ also

$$
\Upsilon^{*}\left(\begin{array}{ccc}
A_{1} & & \\
& \ddots & \\
& & A_{k}
\end{array}\right) \Upsilon=\sum_{j=1}^{k} v_{j}^{*} A_{j} v_{j}
$$

belongs to $\mathbf{A}$.

Background to matrix-convex sets in our context, is given in [13, Sections 2,3], for broader scope literature, see references therein. In particular, from part (I) of [13, Observation 2.2] one can draw the following conclusion with respect to the set $\operatorname{Stein}_{H}(\eta)$ introduced in Eq. 2.2.

Proposition 2.4. For parameters $H \in \mathbf{P}_{n}$ and a scalar $\eta \in(1, \infty]$ the matricial set $\operatorname{Stein}_{H}(\eta)$ in Eq. (2.3) is matrix-convex.

\section{Quantitatively Hyper-Bonded Real Rational functions}

We shall use the following notation for disks in the complex plane

$$
\mathbb{D}(\text { Center, Radius })=\{\lambda \in \mathbb{C}: \text { Radius }>\mid \lambda-\text { Center } \mid\} \quad \begin{aligned}
& \mathrm{C}_{\text {enter }} \in \mathbb{C} \\
& \mathrm{R}_{\text {adius }}>0 .
\end{aligned}
$$

In scalar terminology, a rational function is called Bounded Real if it analytically maps $\mathbb{C}_{R}$ to the closed unit disk $\mathbb{D}(\underbrace{0}_{\text {Center }}, \underbrace{1}_{\text {Radius }})$. Formally, a $m \times m$-valued rational function $G(s)$ is called Bounded Real if

$$
\left(I_{m}-(G(s))^{*} G(s)\right) \in \overline{\mathbf{P}}_{m} \quad \forall s \in \mathbb{C}_{R},
$$

see e.g. [4, Section 2.6] and further analysis in [15, Section 3].

We next "zoom into" this family. To simplify the exposition, we start with scalar rational functions $g(s)$ mapping $\mathbb{C}_{R}$ to a closed sub-unit disk of the form.

$$
\mathbb{D}(\underbrace{0}_{\text {Center }}, \underbrace{\sqrt{\frac{\eta-1}{\eta+1}}}_{\text {Radius }}) \quad \eta \in(1, \infty],
$$

This set of $\eta$-Hyper Bounded Real functions, will be denoted by $\mathcal{H} \mathcal{B}_{\eta}$. For $m \times m$-valued rational functions, we shall describe this family as subsets of functions $G(s)$ in Eq. (3.1),

$$
\left(I_{m}-\frac{\eta+1}{\eta-1}(G(s))^{*} G(s)\right) \in \overline{\mathbf{P}}_{m} \quad \forall s \in \mathbb{C}_{R} .
$$

This condition can be equivalently written as

$$
\sqrt{\frac{\eta-1}{\eta+1}} \leq \sup _{s \in \mathbb{C}_{R}}\|G(s)\|_{2}
$$

In the sequel we shall illustrate the contractive nature of the sets of Hyper-Bounded (and subsequently Hyper-Positive) in several ways, here are scalar samples of $\mathcal{H} \mathcal{B}_{\eta}$ functions.

Example 3.1. Consider a degree one $\mathcal{H B}_{\eta}$ rational function $g(s)$ of the form

$$
g(s)=\sqrt{\frac{\eta-1}{\eta+1}} \cdot \frac{s-a}{s+a} \quad \begin{gathered}
a>0 \\
\eta \in(1, \infty]
\end{gathered}
$$


It maps $\mathbb{C}_{R}$ onto the closed sub-unit disk $\mathbb{D}(\underbrace{0}_{\text {Center }}, \underbrace{\sqrt{\eta-1}}_{\text {Radius }})$, irrespective of the parameter

a. In particular, $g(0)=-\sqrt{\frac{\eta-1}{\eta+1}}, g(i a)=-i \sqrt{\frac{\eta-1}{\eta+1}}, g(\infty)=\sqrt{\frac{\eta-1}{\eta+1}}$. See the Nyquist plot on the left-hand side of Figure 1 .

We now address ourselves to state space realizations of the above rational function $G(s)$. We start with the classical Bounded Real Lemma, see e.g. [4, Section 7.2], [7, Subsection 2.7.3].

Lemma 3.2. Let $G(s)$ be a $m \times m$-valued rational function, with no pole at infinity, of McMillan degree $n$. Let

$$
G(s)=\hat{C}\left(s I_{n}-\hat{A}\right)^{-1} \hat{B}+\hat{D},
$$

be a corresponding minimal realization.

The function $G(s)$ is bounded real, if and only if, there exist a matrix $H$ satisfying

$$
\left.H \in \mathbf{P}_{n} \quad\left(\begin{array}{cc}
-H \hat{A}-\hat{A}^{*} H & -H \hat{B} \\
-\hat{B}^{*} H & I_{m}
\end{array}\right)-\left(\begin{array}{c}
\hat{C}^{*} \\
\hat{D}^{*}
\end{array}\right)\left(\begin{array}{ll}
\hat{C} & \hat{D}
\end{array}\right)\right) \in \overline{\mathbf{P}}_{n+m} .
$$

One can now introduce a quantitative refinement of this classical result.

Lemma 3.3. Let $G(s)$ be a $m \times m$-valued rational function, with no pole at infinity, of McMillan degree $n$. Let

$$
G(s)=\hat{C}\left(s I_{n}-\hat{A}\right)^{-1} \hat{B}+\hat{D},
$$

be a corresponding minimal realization.

We shall say that $m \times m$-valued rational function, belongs to $\mathcal{H B}_{\eta}$ for some $\eta \in(1, \infty]$, i.e. $G(s)$ is quantitatively bounded real in the sense of Eq. (3.2), if and only if, there exist a matrix $H$ satisfying

$$
\left.H \in \mathbf{P}_{n} \quad\left(\begin{array}{cc}
-H \hat{A}-\hat{A}^{*} H & -H \hat{B} \\
-\hat{B}^{*} H & I_{m}
\end{array}\right)-\frac{\eta+1}{\eta-1}\left(\begin{array}{c}
\hat{C}^{*} \\
\hat{D}^{*}
\end{array}\right)\left(\begin{array}{ll}
\hat{C} & \hat{D}
\end{array}\right)\right) \in \overline{\mathbf{P}}_{n+m} .
$$

Moreover, the right-hand side is singular.

The classical case (as in Lemma 3.2) is obtained upon substituting $\eta=\infty$.

In the sequel we shall find it convenient to re-write the condition in Lemma 3.3 in the following quadratic form.

$$
H \in \mathbf{P}_{n} \quad\left(\begin{array}{cc}
\hat{A} & \hat{B} \\
\hat{C} & \hat{D} \\
I_{n} & 0 \\
0 & I_{m}
\end{array}\right)^{*} \underbrace{\left(\begin{array}{cccc}
0 & 0 & -H & 0 \\
0 & \frac{1+\eta}{1-\eta} I_{m} & 0 & 0 \\
-H & 0 & 0 & 0 \\
0 & 0 & 0 & I_{m}
\end{array}\right)}_{W}\left(\begin{array}{cc}
\hat{A} & \hat{B} \\
\hat{C} & \hat{D} \\
I_{n} & 0 \\
0 & I_{m}
\end{array}\right) \in \overline{\mathbf{P}}_{n+m} .
$$

Note that the Hermitian matrix $W$, has exactly $n+m$ positive eigenvalues and $n+m$ negative eigenvalues.

We next resort to the classical Cayley transform.

Definition 3.4. We denote by $\mathcal{C}(M)$ the Cayley transform of a matrix $M \in \mathbb{C}^{n \times n}$, $\hat{M}=\mathcal{C}(M):=\left(I_{n}-M\right)\left(I_{n}+M\right)^{-1}=-I_{n}+2\left(I_{n}+M\right)^{-1} \quad-1 \notin \operatorname{spect}(M)$. 


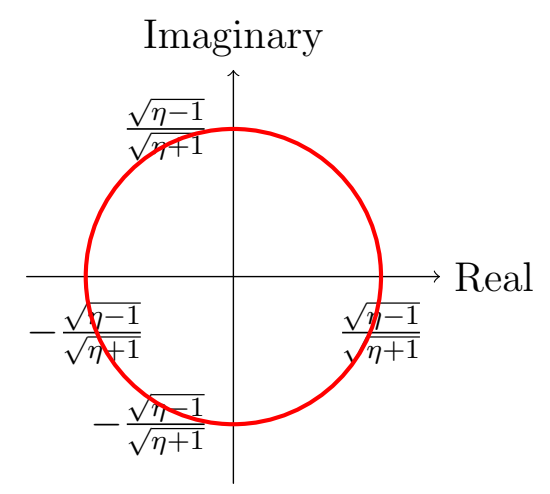

Nyquist plot of $g(s)$ in Eq. 3.4

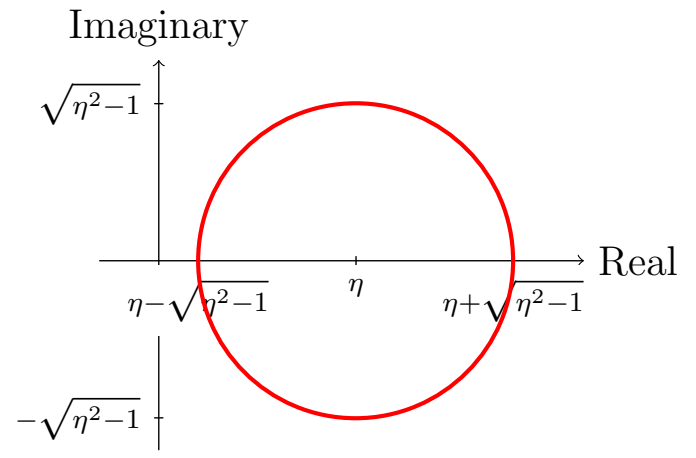

Nyquist plot of $f(s)$ in Eq. (5.4)

Figure 1. $f(s)=\mathcal{C}(g(s))$

As already mentioned, for convenience we follow the (artificial yet intuitive) engineering notational distinction between $M$ (typically with spectrum in $\mathbb{C}_{R}$ ) and $\hat{M}=\mathcal{C}(M)$ (whose spectrum is typically in the unit disk).

Recall that the Cayley transform is involutive in the sense that, whenever well defined,

$$
\mathcal{C}(\mathcal{C}(M))=M
$$

The contractive nature of $\mathcal{H} \mathcal{P}_{\eta}$ functions will be further discussed in Observation 5.8 and through state-space realization presentation, in items (2), (3) of Example 6.4.

Disks of the form of the right-hand side of Figure 11 are studied in details in [14].

\section{QuANTITATIVELY CONTRACTIVE LyAPUNOV INCLUSIONS}

Applying the Cayley transform to the Stein inclusion in Eq. (2.2), yields the Riccati inclusion in Eq. (4.2) below.

Proposition 4.1. For arbitrary $H \in \mathbf{H}_{n}$ and $\eta \in(1, \infty]$ one has that

$$
\mathcal{C}\left(\operatorname{Stein}_{H}(\eta)\right)=\mathbf{L}_{H}(\eta),
$$

where,

$$
\mathbf{L}_{H}(\eta)=\left\{A \in \mathbb{C}^{n \times n}:\left(\begin{array}{c}
A \\
I_{n}
\end{array}\right)^{*}\left(\begin{array}{cc}
-\frac{1}{\eta} H & H \\
H & -\frac{1}{\eta} H
\end{array}\right)\left(\begin{array}{c}
A \\
I_{n}
\end{array}\right)=Q \in \mathbf{P}_{n}\right\}
$$

Remark 4.2. Note that in the Stein equation (2.2), (4.1) and in the Riccati equation (4.2), the parameters $H$ and $\eta$, are indeed the same.

In Eq. 4.2 in particular we denote $\lim _{\eta \rightarrow \infty} \mathbf{L}_{H}(\eta)=\mathbf{L}_{H}$ namely a Lyapunov inclusion,

$$
\mathbf{L}_{H}=\left\{A \in \mathbb{C}^{n \times n}:\left(\begin{array}{c}
A \\
I_{n}
\end{array}\right)^{*}\left(\begin{array}{cc}
0 & H \\
H & 0
\end{array}\right)\left(\begin{array}{c}
A \\
I_{n}
\end{array}\right)=H A+A^{*} H=Q \in \mathbf{P}_{n}\right\} .
$$

For a prescribed $H \in \mathbf{H}_{n}$ one has that,

$$
\infty>\eta>\eta_{1}>1 \quad \Longrightarrow \quad \mathbf{L}_{H}\left(\eta_{1}\right) \subset \mathbf{L}_{H}(\eta) \subset \mathbf{L}_{H}
$$


and each inclusion is strict.

The sets $\overline{\mathbf{L}}_{H}, \mathbf{L}_{H}$ (where $\eta=\infty$ ) were introduced in [10], where it was shown that they are convex cones and closed under inversion. In [13] it was shown that for $H=I_{n}$, these sets are in fact matrix-convex. Here, we colloquially "zoom-in" into this structure to present the main result of this section.

Theorem 4.3. Let $H \in \mathbf{P}_{n}$ and a scalar $\eta \in(1, \infty]$, be prescribed.

(I) Then, the open set $\mathbf{L}_{H}(\eta)$ in Eq. 4.2 is convex and closed under inversion.

(II) For $H \in \mathbf{P}_{n}$ and $\eta=\infty$ the set $\mathbf{L}_{H}$ is a maximal convex set of matrices whose spectrum is in $\mathbb{C}_{R}$.

(III) For $H=I_{n}$, the set $\mathbf{L}_{I_{n}}(\eta)$, is in addition matrix-convex.

Proof : (I) To show convexity, for a pair of $n \times n$ matrices $A_{o}, A_{1}$ and a scalar parameter $\theta \in[0,1]$, consider the following identity,

$$
\begin{gathered}
\underbrace{\theta\left(\begin{array}{c}
A_{1} \\
I_{n}
\end{array}\right)^{*}\left(\begin{array}{cc}
-\frac{1}{\eta} H & H \\
H & -\frac{1}{\eta} H
\end{array}\right)\left(\begin{array}{c}
A_{1} \\
I_{n}
\end{array}\right)}_{Q_{1}}+(1-\theta) \underbrace{\left(\begin{array}{c}
A_{o} \\
I_{n}
\end{array}\right)^{*}\left(\begin{array}{cc}
-\frac{1}{\eta} H & H \\
H & -\frac{1}{\eta} H
\end{array}\right)\left(\begin{array}{c}
A_{o} \\
I_{n}
\end{array}\right)}_{Q_{o}}+\frac{\theta(1-\theta)}{\eta}\left(A_{o}-A_{1}\right)^{*} H\left(A_{o}-A_{1}\right) \\
=\left(\begin{array}{c}
\theta A_{1}+(1-\theta) A_{o} \\
I_{n}
\end{array}\right)^{*}\left(\begin{array}{cc}
-\frac{1}{\eta} H & H \\
H & -\frac{1}{\eta} H
\end{array}\right)\left(\begin{array}{c}
\theta A_{1}+(1-\theta) A_{o} \\
I_{n}
\end{array}\right) .
\end{gathered}
$$

The condition $A_{o}, A_{1} \in \mathbf{L}_{H}(\eta)$ is equivalent to having $Q_{o}, Q_{1} \in \mathbf{P}_{n}$. Since by assumption $H \in \mathbf{P}_{n}$, it implies that $\left(A_{o}-A_{1}\right)^{*} H\left(A_{o}-A_{1}\right) \in \overline{\mathbf{P}}_{n}$ and thus one has that for all $\theta \in[0,1]$, also $\left(\theta A_{1}+(1-\theta) A_{o}\right)$ belongs to $\mathbf{L}_{H}(\eta)$, so this part of the claim is established.

We shall show invertibility in two ways:

(a) Directly: Using Eq. (4.2) note that whenever $A \in \mathbf{L}_{H}(\eta)$ then,

$$
\left(\begin{array}{c}
A^{-1} \\
I_{n}
\end{array}\right)^{*}\left(\begin{array}{cc}
-\frac{1}{\eta} H & H \\
H & -\frac{1}{\eta} H
\end{array}\right)\left(\begin{array}{c}
A^{-1} \\
I_{n}
\end{array}\right)=\left(\begin{array}{c}
I_{n} \\
A^{-1}
\end{array}\right)^{*}\left(\begin{array}{cc}
-\frac{1}{\eta} H & H \\
H & -\frac{1}{\eta} H
\end{array}\right)\left(\begin{array}{c}
I_{n} \\
A^{-1}
\end{array}\right)=\left(A^{-1}\right)^{*} Q\left(A^{-1}\right) \in \mathbf{P}_{n} .
$$

(b) Invertibility can be indirectly deduced by using the relation $\mathcal{C}\left(A^{-1}\right)=-\mathcal{C}(A)$ which is immediate from Definition 3.4, along with Eqs. (2.2) and (2.3).

(II) See e.g. [10, Lemma 3.5].

(III) Matrix convexity for $H=I_{n}$.

First note that one can always complete a given $k n \times n$ isometry $\Upsilon$ to a $k n \times k n$ unitary matrix $U$, i.e. to construct a $k n \times(k-1) n$ matrix $\tilde{U}$ so that

$$
I_{k n}=\underbrace{(\Upsilon \quad \tilde{U}}_{U}) \underbrace{(\Upsilon \quad \tilde{U})^{*}}_{U^{*}}=\Upsilon \Upsilon^{*}+\tilde{U} \tilde{U}^{*}
$$

For a prescribed $\eta \in(1, \infty]$ let now $A_{1}, \ldots, A_{k}$ be in $\mathbf{L}_{I_{n}}(\eta)$, then by Eq. (4.2),

$$
-\frac{1}{\eta} A_{j}^{*} A_{j}+A_{j}^{*}+A_{j}-\frac{1}{\eta} I_{n}=\Delta_{j} \quad \begin{gathered}
\Delta_{j} \in \mathbf{P}_{n} \\
j=1, \ldots, k .
\end{gathered}
$$

We need to show that each matrix of the form

$$
\sum_{j=1}^{k} v_{j}^{*} A_{j} v_{j}=\Upsilon^{*}\left(\begin{array}{ccc}
A_{1} & & \\
& \ddots & \\
& & A_{k}
\end{array}\right) \Upsilon
$$


satisfies the condition in Eq. 4.2.

Indeed,

$$
\begin{aligned}
& -\frac{1}{\eta}\left(\sum_{j=1}^{k} v_{j}^{*} A_{j} v_{j}\right)^{*}\left(\sum_{j=1}^{k} v_{j}^{*} A_{j} v_{j}\right)+\left(\sum_{j=1}^{k} v_{j}^{*} A_{j} v_{j}\right)^{*}+\left(\sum_{j=1}^{k} v_{j}^{*} A_{j} v_{j}\right)-\frac{1}{\eta} I_{n} \\
& =\quad-\frac{1}{\eta}\left(\sum_{j=1}^{k} v_{j}^{*} A_{j} v_{j}\right)^{*}\left(\sum_{j=1}^{k} v_{j}^{*} A_{j} v_{j}\right)+\sum_{j=1}^{k} v_{j}^{*} \underbrace{\left(A_{j}+A_{j}^{*}-\frac{1}{\eta} I_{n}\right)}_{\frac{1}{\eta} A_{j}^{*} A_{j}+\Delta_{j} \text { by Eq.4.4 }} v_{j} \\
& =\quad-\frac{1}{\eta}\left(\sum_{j=1}^{k} v_{j}^{*} A_{j} v_{j}\right)^{*}\left(\sum_{j=1}^{k} v_{j}^{*} A_{j} v_{j}\right)+\sum_{j=1}^{k} v_{j}^{*}\left(\frac{1}{\eta} A_{j}^{*} A_{j}+\Delta_{j}\right) v_{j}
\end{aligned}
$$

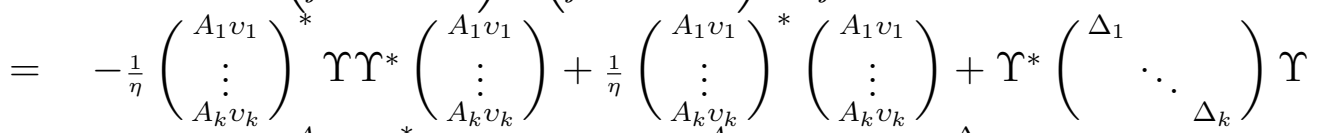

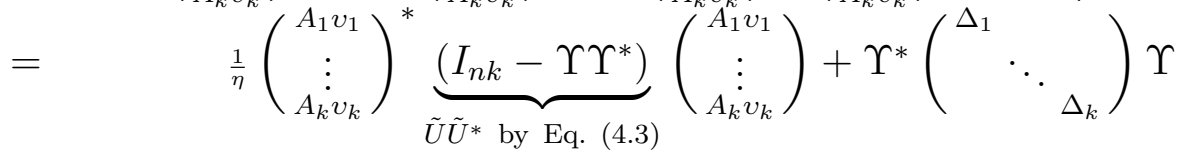

$$
\begin{aligned}
& =\quad \frac{1}{\eta}\left(\begin{array}{c}
A_{1} v_{1} \\
\vdots \\
A_{k} v_{k}
\end{array}\right)^{*} \tilde{U} \tilde{U}^{*}\left(\begin{array}{c}
A_{1} v_{1} \\
\vdots \\
A_{k} v_{k}
\end{array}\right)+\Upsilon^{*}\left(\begin{array}{ccc}
\Delta_{1} & & \\
& \ddots & \\
& & \Delta_{k}
\end{array}\right) \Upsilon .
\end{aligned}
$$

As we have a sum of positive semi-definite matrices, the matrix $\sum_{j=1}^{k} v_{j}^{*} A_{j} v_{j}$ satisfies Eq. (4.2) and thus belongs to $\mathbf{L}_{I_{n}}(\eta)$, so the claim is established.

The above structural properties will be exploited in Corollary 5.5 below, where we address $m \times m$-valued rational functions mapping $\mathbb{C}_{R}$ to

$$
\overline{\mathbf{L}}_{I_{m}}=\left\{A \in \mathbb{C}^{m}: A+A^{*} \in \overline{\mathbf{P}}_{m}\right\}
$$

\section{Quantitatively Hyper-Positive Real Functions}

We start by setting up the framework for positive real rational functions and their subsets.

Definition 5.1. Consider the following families of $m \times m$-valued rational functions $F(s)$.

- The family of functions analytically mapping $\mathbb{C}_{R}$ to $\overline{\mathbf{L}}_{I_{m}}$ is called Positive Real and denoted by $\mathcal{P}$.

- The family of functions $F(s)$ so that $F(s-\epsilon)$ is positive real, for some $\epsilon>0$, is called Strictly Positive Real and denoted by $\mathcal{S P}$.

- The family of functions analytically mapping $\mathbb{C}_{R}$ to $\mathbf{L}_{I_{m}}$ is called Hyper-Positive Real and denoted by $\mathcal{H} \mathcal{P}$.

As a scalar illustration consider the following degree one $\mathcal{P}$ unction

$$
f(s)=d+\frac{b}{s+a} \quad a, d \geq 0 \quad \text { parameters. }
$$

$f \in \mathcal{S P}$ if and only if $a b>0, d \geq 0$ and $f \in \mathcal{H P}$ if and only if $a b d>0$. 
While the sets $\mathcal{P}$ and $\mathcal{S P}$ are classical, see e.g. [8, the name $\mathcal{H} \mathcal{P}$, is less conventional. For instance, in some research circles, see e.g. [8], this family of functions is referred to as "both input and output strictly passive".

Here is a summary of the structure of the set $\mathcal{P}$. For details see [13, Theorem 4.3].

Observation 5.2. Consider the sets of $m \times m$-valued rational functions: $\mathcal{P}, \mathcal{S P}$ and $\mathcal{H} \mathcal{P}$

(i) $\quad \mathcal{H P} \subset \mathcal{S P} \subset \mathcal{P}$ and each inclusion is strict.

(ii) Each of these three sets is a matrix-convex cone and closed under inversion.

(iii) In item (ii), the set $\mathcal{P}$ is maximal in the sense that if a real $m \times m$-valued rational function is not positive real, by taking positive scaling, sums and inversion of this function, along with $F(s) \equiv I_{m}$ (a zero degree positive real function), one can always obtain a function which is no longer analytic in $\mathbb{C}_{R}$.

Recall that in [9] Otto Brune showed that the driving point immittance of a lumped $R-L-C$ electrical network belongs to $\mathcal{P}$ and that an arbitrary positive real rational function can be realized as the driving point immittance of a lumped $R-L-C$ electrical network. For further details see e.g. [4], [6] and [22]. For a recent comprehensive account of circuits describing $\mathcal{P}$ functions of degree two, see [16].

Roughly speaking, $\mathcal{H P}$ functions correspond to the driving point immittance of lumped $R-L-C$ electrical networks with no branch which is puerly reactive (i.e. without a resistive) part.

In the sequel we refine the above definition of $\mathcal{H P}$ functions by introducing a parameter $\eta$, so that

$$
\infty>\eta>\eta_{1}>1 \quad \Longrightarrow \quad \mathcal{H} \mathcal{P}_{\eta_{1}} \subset \mathcal{H} \mathcal{P}_{\eta} \subset \mathcal{H} \mathcal{P}
$$

and each inclusion is strict. Here are the details.

It is well known, see e.g. [4, Example 2.7.1], that the families of Bounded Real and Positive Real rational functions are related through the Cayley transform, i.e.

$$
\mathcal{C}(\mathcal{B})=\mathcal{P}
$$

Following this intuition, we shall similarly describe the set $\mathcal{H} \mathcal{P}_{\eta}$ of Quantitatively HyperPositive Real,

$$
\mathcal{C}\left(\mathcal{H B}_{\eta}\right)=\mathcal{H} \mathcal{P}_{\eta}
$$

Proposition 4.1 relates, through the Cayley transform, the matricial families $\left(\operatorname{Stein}_{H}(\eta)\right)$ and $\mathbf{L}_{H}(\eta)$. In an analogous way, one has the following for matrix-valued, rational $\mathcal{H} \mathcal{P}_{\eta}$ functions.

Observation 5.3. Let $F(s)$ be a $m \times m$-valued rational function and let $\eta, \eta \in(1, \infty]$, be a parameter. The function $F(s)$ belongs to $\mathcal{H P}_{\eta}$, if and only if

$$
\left(\begin{array}{c}
F \\
I
\end{array}\right)^{*}\left(\begin{array}{cc}
-\frac{1}{\eta} I_{n} & I_{n} \\
I_{n} & -\frac{1}{\eta} I_{n}
\end{array}\right)\left(\begin{array}{c}
F \\
I
\end{array}\right) \in \overline{\mathbf{P}}_{m} \quad \forall s \in \mathbb{C}_{R} .
$$

In a way similar to the discussion following Remark 4.2 , taking the limit $\eta \longrightarrow \infty$, the quadratic inclusion in Eq. (5.1) degenerates to a linear inclusion and the set

$$
\mathcal{P}:=\left\{F(s): F+F^{*} \in \overline{\mathbf{P}}_{n} \forall s \in \mathbb{C}_{R}\right\},
$$

of Positive Real functions, is recovered. For more details on this set see [13]. 
Denoting by $\rho(M)$ the spectral radius of a square matrix $M$, one obtains the following equivalent, and technically more convenient, characterization of this set.

Corollary 5.4. Let $F(s)$ be a $m \times m$-valued rational function and let $\eta, \eta \in(1, \infty]$, be a parameter. The function $F(s)$ belongs to $\mathcal{H} \mathcal{P}_{\eta}$, if and only if

$$
\eta \geq \sup _{s \in \mathbb{C}_{R}} \rho\left(\left(F^{*} F+I_{m}\right)\left(F^{*}+F\right)^{-1}\right) .
$$

Proof : From Eq. (5.1) one has the following chain of relations

$$
\begin{array}{clrl}
\left(\left(F+F^{*}\right)-\frac{1}{\eta}\left(F^{*} F+I\right)\right) & \in \overline{\mathbf{P}}_{m} & \forall s \in \mathbb{C}_{R} \\
\left(\eta\left(F^{*}+F\right)-\left(I_{m}+F^{*} F\right)\right) & \in \overline{\mathbf{P}}_{m} & \forall s \in \mathbb{C}_{R} \\
\left(\eta I_{m}-\left(F^{*}+F\right)^{-\frac{1}{2}}\left(I_{m}+F^{*} F\right)\left(F^{*}+F\right)^{-\frac{1}{2}}\right) & \in \overline{\mathbf{P}}_{m} & \forall s \in \mathbb{C}_{R} \\
\eta \geq\left\|\left(F^{*}+F\right)^{-\frac{1}{2}}\left(I_{m}+F^{*} F\right)\left(F^{*}+F\right)^{-\frac{1}{2}}\right\|_{2} & & \forall s \in \mathbb{C}_{R},
\end{array}
$$

which in turn means that

$$
\begin{aligned}
\eta & \geq \sup _{s \in \mathbb{C}_{R}}\left\|\left(F^{*}+F\right)^{-\frac{1}{2}}\left(F^{*} F+I_{m}\right)\left(F^{*}+F\right)^{-\frac{1}{2}}\right\|_{2} \\
& =\sup _{s \in \mathbb{C}_{R}} \rho\left(\left(F^{*}+F\right)^{-\frac{1}{2}}\left(F^{*} F+I_{m}\right)\left(F^{*}+F\right)^{-\frac{1}{2}}\right) \\
& =\sup _{s \in \mathbb{C}_{R}} \rho\left(\left(F^{*} F+I_{m}\right)\left(F^{*}+F\right)^{-1}\right),
\end{aligned}
$$

so Eq. (5.2) is established.

The following alternative description of the set $\mathcal{H} \mathcal{P}_{\eta}$ will be employed in the sequel.

Corollary 5.5. Let $\eta, \eta \in(1, \infty]$, be a parameter. An $m \times m$-valued rational function $F(s)$ belongs to $\mathcal{H} \mathcal{P}_{\eta}$, if and only if, $F(s)$ analytically map $\mathbb{C}_{R}$ to $\mathbf{L}_{I_{m}}(\eta)$, see Eq. (4.2).

Indeed, from Observation 5.3 , one can say that whenever $F(s)$ is an $m \times m$-valued $\mathcal{H} \mathcal{P}_{\eta}$ function, for each point $s_{o} \in \mathbb{C}_{R}$ the matrix $F\left(s_{o}\right)$ belongs to $\mathbf{L}_{I_{m}}(\eta)$.

As an illustration, we first consider a structured, scalar, $\mathcal{H} \mathcal{P}_{\eta}$ function of degree one.

Example 5.6. In scalar terminology, Observation 5.3 may be interpreted as saying that that an $\mathcal{H} \mathcal{P}_{\eta}$ function, maps $\mathbb{C}_{R}$ to a closed disk of the form,

$$
\mathbb{D}(\underbrace{\eta}_{\text {Center }}, \underbrace{\sqrt{\eta^{2}-1}}_{\text {Radius }}), \quad \eta \in(1, \infty] .
$$

As a side remark, we mention that under inversion, this disk is mapped onto itself. For further details see [14, Section 3]

Of particular interest, among the $\mathcal{H} \mathcal{P}_{\eta}$ functions, is the degree one rational function of the form,

$$
f(s)=\eta-\sqrt{\eta^{2}-1}+\frac{2 a \sqrt{\eta^{2}-1}}{s+a} \quad \begin{gathered}
\eta \in(1, \infty] \\
a>0,
\end{gathered}
$$

which passes through the points $f(0)=\eta+\sqrt{\eta^{2}-1}, f(i a)=\eta-i \sqrt{\eta^{2}-1}$, and $f(\infty)=\eta-\sqrt{\eta^{2}-1}$. More generally, this $f(s)$ analytically maps $\mathbb{C}_{R}$ onto the disk in Eq. (5.3), irrespective of the value of the parameter $a$. See the right-hand side of Figure 1. 
Recall that in Eq. (3.4) we considered the degree one $\mathcal{H} \mathcal{B}_{\eta}$ function $g(s)=\sqrt{\frac{\eta-1}{\eta+1}} \frac{s-a}{s+a}$, see the left-hand side of Figure 1. Recall that in fact,

$$
f(s)=\mathcal{C}(g(s)) \quad \text { with } \quad g(s) \text { in Eq. } 3.4 .
$$

As already mentioned following the work of O. Brune, the rational function $f(s)$ in Eq. (5.4) may be realized as the driving point impedance of the simple electrical circuit in Figure 2 .

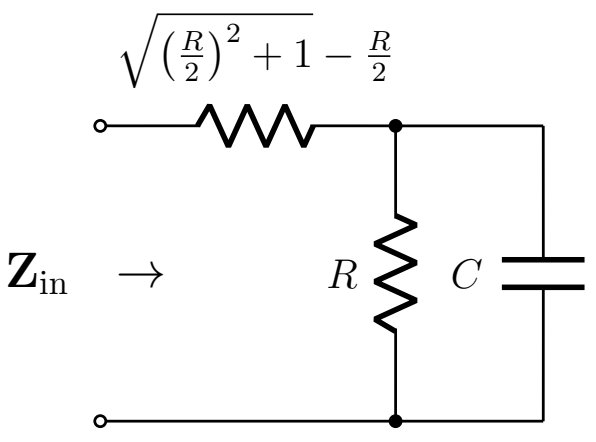

Figure 2. $Z_{\text {in }}=\eta-\sqrt{\eta^{2}-1}+\frac{2 a \sqrt{\eta^{2}-1}}{s+a} \quad C=\frac{1}{a R}$.

In the sequel, we shall relate to $f(s)$ in Eq. (5.4) as a representative of $\mathcal{H} \mathcal{P}_{\eta}$ functions of degree one.

5.1. The Lurie Problem - absolute stability. We have shown that within $\mathbb{C}_{R}$ the novel function set $\mathcal{H} \mathcal{P}_{\eta}$ is disk-contractive. Below we present an application of this observation to the classical Lurie problem (a.k.a. the absolute stability problem). For simplicity of exposition we here present the scalar version. For more information, see e.g. [8, Sections 3.2-3.5], [12, Section 7.1], [17].

Absolute stability problem: Given a a feedback loop as in Figure 3 where $h(s)$ is a rational function with neither pole nor zero at infinity and in the feedback,

$$
\psi=\psi(t, \text { Out })
$$

is an unknown time-dependent, sector-bounded, non-linearity, satisfying

$$
\begin{array}{ll}
(K \cdot \text { Out }-\psi)(\psi-k \cdot \text { Out }) \geq 0 & \forall \text { Out } \\
& \forall t \geq 0 .
\end{array}
$$

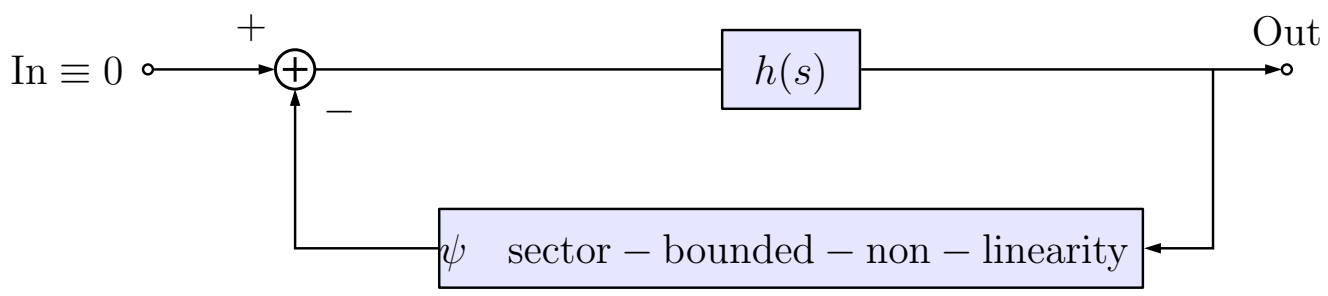

FiguRE 3. Lurie problem feedback loop 
Does there exist $K \geq k$ so that the origin of the overall system is uniformly asymptotically stable for any non-linearity $]^{7} \psi$ satisfying Eq. (5.5).

Find sufficient (and possibly necessary) conditions for that and then obtain various pairs $K, k$ satisfying this requirement.

There are several absolute stability conditions see e.g. [4, Theorem 5.6.3] [12, Sections 7.1], [17], [20, Subsection 2.3.5]. We here refer only to the so called "circle criterion" 8 ,

\section{The Circle Stability Criterion}

Consider the closed-loop system in Figure 3 with Eq. (5.5).

(I) This system is absolutely stable whenever,

$$
(f(h)) \in \mathcal{S P},
$$

for arbitrary rational function $f(s)$, of the form,

$$
f(s)=\frac{1+K s}{1+k s} \quad \text { with } \quad K \geq k .
$$

(II) Whenever $\infty>K \geq k>0$ the above condition can be equivalently formulated as: This system is absolutely stable whenever,

$$
(f(h)) \in \mathcal{S P},
$$

for arbitrary rational function $f(s)$, of the form,

$$
f(s)=\left(\eta-\sqrt{\eta^{2}-1}\right)+\frac{2 a \sqrt{\eta^{2}-1}}{s+a} \text { with } \begin{array}{rlr}
\eta & = & \frac{1}{2}\left(\sqrt{\frac{K}{k}}+\sqrt{\frac{k}{K}}\right) \\
a & = & \frac{1}{K} .
\end{array}
$$

Indeed, the statement in (I) is classical, see e.g. [4, Theorem 5.6.2], [12, Theorem 7.1], [19, Sections 2.11, 3.10], [20, Proposition 3.6].

As for (II), recall that by item (ii) of Observation 5.2, the set $\mathcal{S P}$ is a cone, and closed under inversion. Hence,

$$
f(h(s)) \in \mathcal{S P} \quad \Longrightarrow \quad \beta f(h(s))^{-1} \in \mathcal{S P} \quad \forall \beta>0 .
$$

Now, restricting the discussion to $\infty>K \geq k>0$ means that one can take

$$
f(s)=\beta\left(\frac{1+K s}{1+k s}\right)^{-1}=\left.\beta \frac{1+k s}{1+K s}\right|_{\beta=\frac{\sqrt{K}}{\sqrt{k}}}=\frac{\sqrt{k}}{\sqrt{K}}+\frac{\left(\frac{\sqrt{K}}{\sqrt{k}}-\frac{\sqrt{k}}{\sqrt{K}}\right) \overbrace{\frac{1}{K}}^{a+\underbrace{\frac{1}{K}}_{a}},}{a},
$$

which is of the form of Eqs. (5.4) and (5.8).

To summarize, the Circle Stability criterion may be formulated in terms of a degree one rational function in $\mathcal{H} \mathcal{P}_{\eta}$ as in (5.8).

\footnotetext{
${ }^{7}$ For $K=k$, this is a question on stability of linear-time-invariant system, so we actually focus ourselves on the case $K>k$.

8 The graphical interpretation leading to the name "circle criterion", is beyond the scope of this work.
} 
5.2. Structure of $\mathcal{H} \mathcal{P}_{\eta}$. Following Eqs. (5.6) and (5.8), we start by showing that the set $\mathcal{H} \mathcal{P}_{\eta}$ is contractive in the sense of composition.

In [18] F.M. Reza, in the framework of scalar positive real rational functions, coined the term "power dominance": A function $f_{1}(s)$ "dominates" $f_{2}(s)$ whenever the Nyquist plot of $f_{2}(s)$ is contained in the Nyquist plot $f_{1}(s)$. Furthermore, under certain conditions one can find a third function $h(s)$ so that these functions are related through composition,

$$
f_{2}(s)=f_{1}(h(s)) .
$$

Reza, subsequently applied this idea to the construction of $R-L-C$ circuits.

Example 5.7. We here illustrate F.M. Reza's "power dominance" in the framework of $\mathcal{H} \mathcal{P}_{\eta}$ functions. In particular, as the function $f(s)$ in Eq. (5.8),

$$
f(s)=\left(\eta-\sqrt{\eta^{2}-1}\right)+\frac{2 a \sqrt{\eta^{2}-1}}{s+a} \quad a>0,
$$

analytically maps $\mathbb{C}_{R}$ onto $\mathbb{D}(\underbrace{\eta}_{\text {Center }}, \underbrace{\sqrt{\eta^{2}-1}}_{\text {Radius }})$, this $f(s)$, dominates all functions in $\mathcal{H} \mathcal{P}_{\eta}$.

See the right-hand side of Figure 1 and for $\eta=\sqrt{2}$, the red curve in Figure 4 .

(1) As was already pointed out, a degree one rational $\mathcal{H} \mathcal{P}$ function is of the form

$$
\phi(s)=d+\frac{b}{s+a} \quad a b d>0 .
$$

This $\phi(s)$ analytically maps $\mathbb{C}_{R}$ to a closed disk $\mathbb{D}(\underbrace{d+\frac{b}{2 a}+0 i}_{\text {Center }}, \underbrace{\frac{b}{2 a}}_{\text {Radius }})$ Substitution in Eq. (5.2) reveals that the function in Eq. (5.9) in fact belongs to $\mathcal{H} \mathcal{P}_{\eta}$ where,

$$
\eta=\left\{\begin{array}{cc}
\frac{1}{2}\left(d+\frac{1}{d}\right) & d \in\left(0, \frac{\sqrt{b^{2}+4 a^{2}}-b}{2 a}\right) \\
\frac{1}{2}\left(\frac{d a+b}{a}+\frac{a}{d a+b}\right) & d>\frac{\sqrt{b^{2}+4 a^{2}}-b}{2 a} .
\end{array}\right.
$$

As already mentioned, all functions of the form of $\phi(s)$ in Eq. (5.9) are dominated by the above $f(s)$.

(2) Consider now a degree two $\mathcal{H} \mathcal{P}$ rational function

$$
\phi(s)=\frac{\sqrt{2} a^{2}}{(s+a)^{2}}+\frac{1}{\sqrt{2}} \quad a>0 .
$$

Note that $\phi(0)=\frac{3}{\sqrt{2}}, \phi( \pm i a)=\frac{1}{\sqrt{2}}(1 \mp i), \phi( \pm \sqrt{3} a i)=\frac{3}{4 \sqrt{2}}(1 \mp i)$ and $\phi(\infty)=\frac{1}{\sqrt{2}}$. In fact,

$$
\sup _{s \in \mathbb{C}_{R}} \frac{\phi^{*} \phi+1}{\phi^{*}+\phi}=\left.\frac{\phi^{*} \phi+1}{\phi^{*}+\phi}\right|_{s= \pm i a}=\frac{2}{\frac{2}{\sqrt{2}}}=\sqrt{2},
$$

so from Eq. 5.2 one can conclude that $\phi(s)$ belongs to $\mathcal{H} \mathcal{P}_{\sqrt{2}}$, see the blue curve in Figure 4. As already pointed out in the beginning of this example, this $\phi(s)$ is dominated by,

$$
f(s)=\sqrt{2}-1+\frac{2 a}{s+1} \quad a>0,
$$

see the red curve in Figure 4. In particular note that $f(s)_{\left.\right|_{s= \pm i a(\sqrt{2}+1)}}=\frac{1}{\sqrt{2}}(1 \mp i)=$ $\left.\phi(s)\right|_{s= \pm i a}$. 


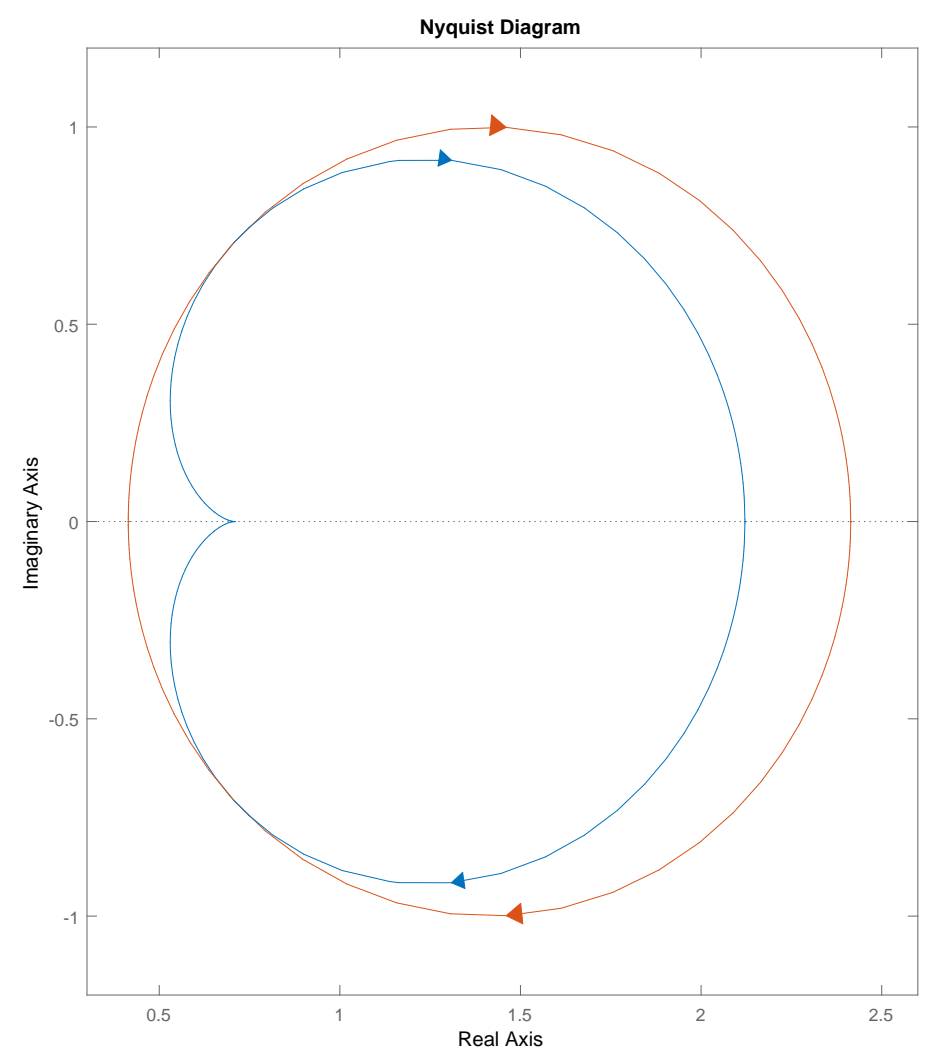

Figure 4. Nyquist plots of $f(s)$ (red) and of $\phi(s)$ (blue), from Eqs. 5.4 (5.10), respectively

Next, we extend Reza's approach to a matrix-valued setup.

Observation 5.8. Let $F(s)$ be a $m \times m$-valued rational function in $\mathcal{H} \mathcal{P}_{\eta}$ for some $\eta \in(1, \infty]$. Let $h(s)$ be a scalar rational function in $\mathcal{S P}$. Then the composed $m \times m$-valued rational function

$$
F(h(s))
$$

belongs to $\mathcal{H P}_{\eta_{1}}$ for some

$$
\eta_{1} \in(1, \eta] .
$$

Indeed, as by definition $h(s)$ maps $\mathbb{C}_{R}$ into $\mathbb{C}_{R}$, one has that,

$$
\begin{aligned}
\eta=\sup _{s \in \mathbb{C}_{R}} \rho\left(\left(F(s)^{*} F(s)+I_{m}\right)\left(F(s)^{*}+F(s)\right)^{-1}\right) & \geq \sup _{s \in h\left(\mathbb{C}_{R}\right)} \rho\left(\left(F(s)^{*} F(s)+I_{m}\right)\left(F(s)^{*}+F(s)\right)^{-1}\right) \\
& =\sup _{s \in \mathbb{C}_{R}} \rho\left(\left(F(h)^{*} F(h)+I_{m}\right)\left(F(h)^{*}+F(h)\right)^{-1}\right)=\eta_{1} .
\end{aligned}
$$


We further explore the structure of the set $\mathcal{H} \mathcal{P}_{\eta}$. For perspective recall that in [13] it was shown that the set of positive real functions is matrix-convex cone, closed under inversion.

Proposition 5.9. For a given $\eta \in(1, \infty]$, the set $\mathcal{H} \mathcal{P}_{\eta}$ is matrix-convex. It is also closed under inversion, i.e. whenever a rational function $F(s)$ is in $\mathcal{H} \mathcal{P}_{\eta}$, then $(F(s))^{-1}$ is well defined and belongs to $\mathcal{H P}_{\eta}$, with the same $\eta$.

Proof : Matrix-convexity. Recall that by item (II) of Theorem 4.3 the set $\mathbf{L}_{I_{m}}(\eta$ is matrix-convex. Combining this fact along with with the alternative description of the set $\mathcal{H} \mathcal{P}_{\eta}$ in Corollary 5.5, establishes the claim.

We shall show invertibility in two ways.

(i) Direct approach. Using Eq. (5.2) one has that,

$$
\begin{aligned}
& \eta(F)=\quad \sup _{s \in \mathbb{C}_{R}} \rho\left(\left(F^{*} F+I_{m}\right)\left(F^{*}+F\right)^{-1}\right) \\
& =\sup _{s \in \mathbb{C}_{R}} \rho(\underbrace{F^{*}\left(\left(F^{*}\right)^{-1} F^{-1}+I_{m}\right) F}_{\left(F^{*} F+I_{m}\right)} \underbrace{F^{-1}\left(\left(F^{*}\right)^{-1}+F^{-1}\right)^{-1}\left(F^{*}\right)^{-1}}_{\left(F^{*}+F\right)^{-1}}) \\
& =\quad \sup _{s \in \mathbb{C}_{R}} \rho\left(F^{*}\left(\left(F^{*}\right)^{-1} F^{-1}+I_{m}\right)\left(\left(F^{*}\right)^{-1}+F^{-1}\right)^{-1}\left(F^{*}\right)^{-1}\right) \\
& =\quad \sup _{s \in \mathbb{C}_{R}} \rho\left(\left(\left(F^{-1}\right)^{*} F^{-1}+I_{m}\right)\left(\left(F^{-1}\right)^{*}+F^{-1}\right)^{-1}\right) \quad=\eta\left(F^{-1}\right) .
\end{aligned}
$$

(ii) Indirect (yet easy) approach. Applying Definition 3.4 of the Cayley transform, to a function $F(s)$, it is easy to verify that

$$
\mathcal{C}\left((F)^{-1}\right)=-\mathcal{C}(F) .
$$

Thus clearly, for any $s \in \mathbb{C}$ where $F(s)$ is analytic, it holds that

$$
\|\mathcal{C}(F)\|_{2}=\|-\mathcal{C}(F)\|_{2}=\left\|\mathcal{C}\left(F^{-1}\right)\right\|_{2} .
$$

This implies that

$$
\sup _{s \in \mathbb{C}_{R}}\|\mathcal{C}(F(s))\|_{2}=\sup _{s \in \mathbb{C}_{R}}\left\|\mathcal{C}\left(F(s)^{-1}\right)\right\|_{2},
$$

Assuming that $F(s)$ belongs to $\mathcal{H} \mathcal{P}_{\eta}$, by Observation 5.3 it follows that this is equivalent to,

$$
\frac{\eta-1}{\eta+1}=\sup _{s \in \mathbb{C}_{R}}\|\mathcal{C}(F(s))\|_{2}=\sup _{s \in \mathbb{C}_{R}}\left\|\mathcal{C}\left(F(s)^{-1}\right)\right\|_{2},
$$

so by Eq. (3.3), the claim is established.

\section{Kalman-Yakubovich-Popov Lemma for $\mathcal{H} \mathcal{P}_{\eta}$ Functions}

A state-space realization of a given $m \times m$-valued rational function $F(s)$, with no pole at infinity, is given by

$$
F(s)=C\left(s I_{n}-A\right)^{-1} B+D \quad R_{F}=\left(\begin{array}{c|c}
A & B \\
\hline C & D
\end{array}\right) .
$$

The $(n+m) \times(n+m)$ array $R_{F}$ was introduced by H.H. Rosenbrock, see [19]. In general, $n$ is larger or equal to the McMillan degree of $F(s)$. Equality means that the realization is minimal. 
In the first subsection, we take advantage of the dual nature of $R_{F}$ as an array and as a matrix.

\subsection{K-Y-P for Positive functions.}

Theorem 6.1. Let $F(s)$ be a $m \times m$-valued rational function with no pole at infinity and let $R_{F}$ in (6.1) be a corresponding $(n+m) \times(n+m)$ state-space realization.

For some $H \in \mathbf{P}_{n}$ consider the following Lyapunov equation.

$$
\left(\begin{array}{cc}
-H & 0 \\
0 & I_{m}
\end{array}\right)\left(\begin{array}{cc}
A & B \\
C & D
\end{array}\right)+\left(\begin{array}{cc}
A & B \\
C & D
\end{array}\right)^{*}\left(\begin{array}{cc}
-H & 0 \\
0 & I_{m}
\end{array}\right)=Q .
$$

(i) If in Eq. (6.2) $Q \in \overline{\mathbf{P}}_{n+m}$ then $F(s)$ is a $\mathcal{P}$ function.

(ii) If in Eq. (6.2) $Q=Q_{1}+\left(\begin{array}{cc}\Delta & 0 \\ 0 & 0\end{array}\right)$ with $Q_{1} \in \overline{\mathbf{P}}_{n+m}$ and $\Delta \in \mathbf{P}_{n}$, then $F(s)$ is a $\mathcal{S P}$ function.

(iii) If in Eq. 6.2) $Q \in \mathbf{P}_{n+m}$, then $F(s)$ is a $\mathcal{H P}$ function.

In each of the three above statements, if the realization in Eq. (6.1) is minimal, i.e. $n$ is the McMillan degree, then the converse is true as well.

Proof : (i) Up to a change of coordinates one can substitute in Eq. (6.2) $H=I_{n}$ and thus,

$$
Q=\left(\begin{array}{cc}
-I_{n} & 0 \\
0 & I_{m}
\end{array}\right) \underbrace{\left(\begin{array}{ll}
A & B \\
C & D
\end{array}\right)}_{R_{F}}+\underbrace{\left(\begin{array}{cc}
A & B \\
C & D
\end{array}\right)^{*}}_{R_{F}^{*}}\left(\begin{array}{rr}
-I_{n} & 0 \\
0 & I_{m}
\end{array}\right)=\left(\begin{array}{rr}
-A & -B \\
C & D
\end{array}\right)\left(\begin{array}{rr}
-A & -B \\
C & D
\end{array}\right)^{*}
$$

Next note that for all $s \in \mathbb{C}$,

$$
\begin{aligned}
\hat{Q}(s):=\left(\begin{array}{cc}
2 \operatorname{Re}(s) I_{n} & 0 \\
0 & 0
\end{array}\right)+Q & =\left(\begin{array}{rr}
s I_{n} & 0 \\
0 & 0
\end{array}\right)+\left(\begin{array}{rr}
-A & -B \\
C & D
\end{array}\right)+\left(\begin{array}{rr}
s I_{n} & 0 \\
0 & 0
\end{array}\right)^{*}+\left(\begin{array}{rr}
-A & -B \\
C & D
\end{array}\right)^{*} \\
& =\left(\begin{array}{rr}
s I_{n}-A & -B \\
C & D
\end{array}\right)+\left(\begin{array}{rr}
s I_{n}-A & -B \\
C & D
\end{array}\right)^{*} .
\end{aligned}
$$

Now,

$$
\left(\begin{array}{c}
\left(\left(C\left(A-s I_{n}\right)^{-1}\right)^{*}\right. \\
I_{m}
\end{array}\right)^{*} \hat{Q}(s)\left(\begin{array}{c}
\left(\left(C\left(A-s I_{n}\right)^{-1}\right)^{*}\right. \\
I_{m}
\end{array}\right)=\underbrace{C\left(s I_{n}-A\right)^{-1} B+D}_{F(s) \text { in Eq. 6.1 }}+\underbrace{\left(C\left(s I_{n}-A\right)^{-1} B+D\right)^{*}}_{(F(s))^{*} \text { in Eq. 6.1] }}
$$

and if $Q \in \overline{\mathbf{P}}_{n+m}$ (or $Q \in \mathbf{P}_{n+m}$ ) then for all $s \in \mathbb{C}_{R}$ one has that $\hat{Q}(s) \in \overline{\mathbf{P}}_{n+m}$ (or $\hat{Q}(s) \in \mathbf{P}_{n+m}$ ) and then $F(s) \in \overline{\mathbf{L}}_{I_{m}}$ (or $F(s) \in \mathbf{L}_{I_{m}}$ ) respectively. Hence items (i) and (iii) are established.

(ii) By definitions $F \in \mathcal{S P}$ means that for some $\epsilon>0$ one has that $C\left(s I_{n}-\left(A+\epsilon I_{n}\right)\right)^{-1} B+D$ is positive real. Namely, by item (i), for some $H \in \mathbf{P}_{n}$,

$$
\left(\begin{array}{cc}
-H & 0 \\
0 & I_{m}
\end{array}\right)\left(\begin{array}{cc}
A+\epsilon I_{n} & B \\
C & D
\end{array}\right)+\left(\begin{array}{cc}
A+\epsilon I_{n} & B \\
C & D
\end{array}\right)^{*}\left(\begin{array}{cc}
-H & 0 \\
0 & I_{m}
\end{array}\right)=Q \in \overline{\mathbf{P}}_{n+m}
$$

Then,

$$
\left(\begin{array}{cc}
-H & 0 \\
0 & I_{m}
\end{array}\right)\left(\begin{array}{cc}
A & B \\
C & D
\end{array}\right)+\left(\begin{array}{cc}
A & B \\
C & D
\end{array}\right)^{*}\left(\begin{array}{cc}
-H & 0 \\
0 & I_{m}
\end{array}\right)=\underbrace{Q}_{\in \overline{\mathbf{P}}_{n+m}}+\left(\begin{array}{cc}
2 \epsilon H & 0 \\
0 & 0
\end{array}\right)
$$

so taking $\left(2\|H\|_{2}\left\|\Delta^{-1}\right\|_{2}\right)^{-1} \geq \epsilon$ completes the construction.

There are numerous proofs for variants of Theorem 6.1, see e.g. [1], [4, Chapter 5], [12, Lemma 6.3, Appendix C.12]. The formulation in Eq. (6.2) (introduced in [11]) enables one to obtain the above simple proof. 
In [13, Section 5] we advance in manipulating $R_{F}$ in Eq. (6.1) as a matrix, and consider families of rational functions whose corresponding realization arrays/matrices $R_{F}$, form a convex cone, closed under inversion.

As a consequence of Theorem 6.1, we have the following.

Observation 6.2. Let $F(s)$ be a $m \times m$-valued rational function. Then $F \in \mathcal{H} \mathcal{P}$ if and only if, $F \in \mathcal{S P}$ and in addition, $\lim _{s \rightarrow \infty} F(s)$ exists and belongs to $\mathbf{L}_{I_{m}}$.

Proof : : Following Theorem 6.1, we actually need to show that for a given $H \in \mathbf{P}_{n}$ in Eq. (6.2), having on the right-hand side $Q \in \mathbf{P}_{n+m}$ is equivalent to

$$
\left(\begin{array}{cc}
-H & 0 \\
0 & I_{m}
\end{array}\right)\left(\begin{array}{cc}
A+\epsilon I_{n} & B \\
C & D-\delta I_{m}
\end{array}\right)+\left(\begin{array}{cc}
A+\epsilon I_{n} & B \\
C & D-\delta I_{m}
\end{array}\right)^{*}\left(\begin{array}{cc}
-H & 0 \\
0 & I_{m}
\end{array}\right)=\hat{Q} \quad \hat{Q} \in \overline{\mathbf{P}}_{n+m},
$$

for some $\epsilon, \delta>0$. Indeed, comparison yields

$$
\underbrace{Q}_{\in \mathbf{P}_{n+m}}=\underbrace{\hat{Q}}_{\in \overline{\mathbf{P}}_{n+m}}+2\left(\begin{array}{cc}
\epsilon H & 0 \\
0 & \delta I_{m}
\end{array}\right),
$$

so having $\hat{Q} \in \overline{\mathbf{P}}_{n+m}$ implies that $Q \in \mathbf{P}_{n+m}$.

Conversely, by assumption

$$
\beta:=\frac{1}{2} \min _{j=1, \ldots, n+m} \lambda_{j}(Q),
$$

is positive. Thus taking,

$$
\epsilon \leq \beta\|H\|_{2}^{-1} \quad \text { and } \quad \delta \leq \beta,
$$

guaranties that indeed $\hat{Q} \in \overline{\mathbf{P}}_{n+m}$.

The above reasoning guarantees that $\delta>0$ and thus indeed, $D \in \mathbf{L}_{I_{m}}$, so the claim is established.

6.2. K-Y-P for Hyper-Positive functions. Next we consider $m \times m$-valued rational function $F(s)$ assuming it has no pole at infinity, and is of McMillan degree $n$. Thus, it admits a state space realization

$$
R_{F}=\left(\begin{array}{l|l}
A & B \\
\hline C & D
\end{array}\right) .
$$

Recall that applying the Cayley transform (see Definition 3.4) to $F(s)$ means that whenever $-1 \notin \operatorname{spect} .(D)$,

$$
R_{\mathcal{C}(F)}=R_{2\left(F+I_{m}\right)^{-1}-I_{m}}=\left(\begin{array}{l|l}
\overbrace{A-B\left(I_{m}+D\right)^{-1} C}^{\hat{A}} & \overbrace{-\sqrt{2} B\left(I_{m}+D\right)^{-1}}^{\hat{B}} \\
\hline \underbrace{\sqrt{2}\left(I_{m}+D\right)^{-1} C}_{\hat{C}} & \underbrace{\left(I_{m}+D\right)^{-1}\left(I_{m}-D\right)}_{\hat{D}}
\end{array}\right)
$$

Substituting Eq. 6.5 in Eq. (3.5) yields the main result of this subsection.

Theorem 6.3. Let $F(s)$ be a $m \times m$-valued rational function with no pole at infinity of McMillan degree $n$ whose minimal realization is given in Eq. (6.4).

For some $\eta \in(1, \infty]$, the function $F(s)$ belongs to the class $\mathcal{H} \mathcal{P}_{\eta}$, if and only if, there exists a matrix $H$ satisfying,

$$
H \in \mathbf{P}_{n},
$$


and

$$
\left(\begin{array}{cc}
A-B\left(I_{m}+D\right)^{-1} C & -\sqrt{2} B\left(I_{m}+D\right)^{-1} \\
\sqrt{2}\left(I_{m}+D\right)^{-1} C & \left(I_{m}+D\right)^{-1}\left(I_{m}-D\right) \\
I_{n} & 0 \\
0 & I_{m}
\end{array}\right) \underbrace{\left(\begin{array}{cccc}
0 & 0 & -H & 0 \\
0 & \frac{1+\eta}{1-\eta} I_{m} & 0 & 0 \\
-H & 0 & 0 & 0 \\
0 & 0 & 0 & I_{m}
\end{array}\right)}_{W}\left(\begin{array}{cc}
A-B\left(I_{m}+D\right)^{-1} C & -\sqrt{2} B\left(I_{m}+D\right)^{-1} \\
\sqrt{2}\left(I_{m}+D\right)^{-1} C & \left(I_{m}+D\right)^{-1}\left(I_{m}-D\right) \\
I_{n} & 0 \\
0 & I_{m}
\end{array}\right) \in \overline{\mathbf{P}}_{n+m}
$$

As already mentioned, the Hermitian matrix $W$, has $n+m$ positive and $n+m$ negative eigenvalues.

We next illustrate the quantitative essence of Theorem 6.3 .

Example 6.4. Substituting in Eq. (5.4) $\eta=\frac{5}{3}$ and $a=\frac{1}{9}$, one obtains the degree one $\mathcal{H} \mathcal{P}_{\frac{5}{3}}$ function $f(s)=\frac{1}{3}+\frac{\frac{8}{27}}{s+\frac{1}{9}}=\frac{\frac{1}{3}(s+1)}{s+\frac{1}{9}}$. Recall now that

$$
f_{1}(s)=\left(\frac{1}{2}\left(f(s)+\frac{1}{f(s)}\right)\right)^{-1}=\frac{3}{5}+\frac{\frac{32}{75} s}{s^{2}+\frac{2}{5} s+\frac{1}{9}}=\frac{3\left(s^{2}+\frac{10}{9} s+\frac{1}{9}\right)}{5\left(s^{2}+\frac{2}{5} s+\frac{1}{9}\right)},
$$

is a degree two function in $\mathcal{H} \mathcal{P}_{\eta_{1}}$ where

$$
\eta_{1}=\frac{1}{2}\left(\eta+\frac{1}{\eta}\right)=\frac{1}{2}\left(\frac{5}{3}+\frac{3}{5}\right)=\frac{17}{15} .
$$

In particular, $f_{1}(0)=\frac{3}{5}, f_{1}\left( \pm \frac{i}{3}\right)=\frac{5}{3}$, and $f_{1}(\infty)=\frac{3}{5}$.

As a side remark, note that applying the Cayley transform here yields the corresponding $\mathcal{H B}_{\eta}$ functions, $\mathcal{C}(f(s))=g(s)=\frac{1}{2} \cdot \frac{s-\frac{1}{3}}{s+\frac{1}{3}}$ and $\mathcal{C}\left(f_{1}(s)\right)=(g(s))^{2}=g_{1}(s)=\frac{1}{4} \frac{\left(s-\frac{1}{3}\right)^{2}}{\left(s+\frac{1}{3}\right)^{2}}$.

Now, a balanced realization array of $f_{1}(s)$ in Eq. (6.6) is given by,

$$
R_{f_{1}}=\frac{1}{5}\left(\begin{array}{rr|r}
-1 & -\frac{4}{3} & \frac{4}{\sqrt{6}} \\
\frac{4}{3} & -1 & \frac{8}{\sqrt{6}} \\
\hline \frac{8}{\sqrt{6}} & \frac{4}{\sqrt{6}} & 3
\end{array}\right) .
$$

As before, from the classical K-Y-P Lemma one can (only qualitatively) conclude that $f_{1}(s)$ in Eq. 6.6 is Hyper-Positive. Indeed, taking in Eq. 6.2,

$$
H=I_{2},
$$

yields the following right-hand side,

$$
Q=\frac{2}{5}\left(\begin{array}{ccc}
1 & 0 & \frac{2}{\sqrt{6}} \\
0 & 1 & -\frac{2}{\sqrt{6}} \\
\frac{2}{\sqrt{6}} & -\frac{2}{\sqrt{6}} & 3
\end{array}\right),
$$

which is positive definite.

To quantitatively examine this function, we resort to Theorem 6.3. Following Eq. 6.5), a minimal realization of $\mathcal{C}\left(f_{1}\right)$, the image of this $f_{1}(s)$ under the Cayley transform, is,

$$
R_{\mathcal{C}\left(f_{1}\right)}=\left(\begin{array}{rr|c}
-\frac{1}{3} & -\frac{1}{3} & -\frac{1}{2 \sqrt{3}} \\
0 & -\frac{1}{3} & -\frac{1}{\sqrt{3}} \\
\hline \frac{1}{\sqrt{3}} & \frac{1}{2 \sqrt{3}} & \frac{1}{4}
\end{array}\right) .
$$

Now taking in Lemma 3.3 (or in Theorem 6.3)

$$
\eta_{1}=\frac{17}{15} \text { and } H=\left(\begin{array}{ll}
8 & 0 \\
0 & 2
\end{array}\right) \text {, }
$$

yields that the right-hand side of Eq. (3.5) (or of the quadratic inclusion in Theorem 6.3) is $0_{3 \times 3}$, so indeed $f_{1} \in \mathcal{H} \mathcal{P}_{\frac{17}{15}}$. 


\section{REFERENCES}

[1] D. Alpay and I. Lewkowicz, "The Positive Real Lemma and Construction of all Realizations of Generalized Positive Rational Functions", Systems and Control Letters, Vol. 60, pp. 985-993, 2011.

[2] D. Alpay and I. Lewkowicz, "Composition of Rational Functions: State-space Realization and Applications", to appear in Linear Algebra and its Applications. See arXiv:1807.01753.

[3] D. Alpay and I. Lewkowicz, "Realization of Tensor-Product and of Tensor-Factorization of Rational Functions", Quantum Studies: Mathematics and Foundations, Vol. 6, pp. 269-278, 2019.

[4] B.D.O. Anderson and S. Vongpanitlerd, Networks Analysis and Synthesis, A Modern Systems Theory Approach, Prentice-Hall, New Jersey, 1973.

[5] T. Ando, "Sets of Matrices with Common Stein Solutions and H-contractions, Linear Algebra and its Application, Vol. 383, pp. 49-64, 2004.

[6] V. Belevich, Classical Network Theory, Holden Day, San-Francisco, 1968.

[7] S. Boyd, L. El-Ghaoui, E. Ferron and V. Balakrishnan, Linear Matrix Inequalities in Systems and Control Theory, SIAM books, 1994.

[8] B. Brogliato, R. Lozano, B. Maschke and O. Egeland, Dissipative Systems Analysis and Control: Theory and Applications, Second Edition, Upper Saddle River, New-York, Springer Verlag, 2007.

[9] O. Brune, "Synthesis of a Finite Two Terminal Network whose Driving Point Impedance is a Prescribed Function of Frequency", Journal of Mathematical Physics, Vol. 10, pp. 191-236, 1931.

[10] N. Cohen and I. Lewkowicz, "Convex Invertible Cones and the Lyapunov Equation", Linear Algebra and its Applications, Vol. 250, pp. 265-286, 1997.

[11] B. Dickinson, Ph. Delsarte, Y. Genin and Y. Kamp, "Minimal Realization of Pseudo Positive and Pseudo Bounded Real Rational Matrices, IEEE trans. Circuits and Systems, Vol. 32, pp. 603-605, 1985.

[12] H.K. Khalil, Nonlinear Systems, $3^{\text {rd }}$ edition, Pearson Education, NJ, USA, 2000.

[13] I. Lewkowicz, Passive Linear Systems Matrix-convex Invertible Cones Point of View, a manuscript.

[14] I. Lewkowicz, Matrix Sign Function Iterations - Geometric Point of View, a manuscript.

[15] I. Lewkowicz, Discrete-Time Passive Linear Systems - Multiplicative Matrix-Convex Sets Point of View, a manuscript.

[16] A. Morelli and M.C. Smith, Passive Network Synthesis: An Approach to Classification no. DC33 in Advances in Design and Control series by SIAM, 2019.

[17] V.M. Popov, Hyper-stability of Control Systems, Springer 1973.

[18] F.M. Reza, "The Concept of Power Dominant Systems", Lecture Notes in Control and Information Sciences Vol. 58, pp. 787-795, Springer 1984.

[19] H.H. Rosenbrock, Computer-Aided Control System Design, Academic Press, 1974.

[20] R. Sepulchre, M. Janković and P.V. Kokotović, Constructive Nonlinear Control, Communication and Control Engineering series, Springer, 1996.

[21] J.C. Willems, "Realization of Systems with Internal Passivity and Symmetry Constraints", Journal of the Franklin Institute, Vol. 301, pp. 605-621, 1976.

[22] M. R. Wohlers, Lumped and Distributed Passive Networks, Acad. Press 1969.

(DA) Faculty of Mathematics, Physics, and Computation, Schmidt College of Science and Technology, Chapman University, One University Drive Orange, California 92866, USA

E-mail address: alpay@chapman.edu

(IL) School of Electrical and Computer Engineering, Ben-Gurion University of the Negev, P.O.B. 653, Beer-Sheva, 84105, Israel

E-mail address: izchak@bgu.ac.il 